\title{
A LIMIT THEOREM FOR THE TAILS OF DISCRETE INFINITELY DIVISIBLE LAWS WITH APPLICATIONS TO FLUCTUATION THEORY
}

\author{
PAUL EMBRECHTS and JOHN HAWKES
}

(Received 12 December 1980, revised 22 April 1981)

Communicated by R. L. Tweedie

\begin{abstract}
Suppose that $\left(p_{n}\right)$ is an infinitely divisible distribution on the non-negative integers having Lévy measure $\left(\nu_{n}\right)$. In this paper we derive a necessary and sufficient condition for the existence of the limit $\lim _{n \rightarrow \infty} p_{n} / \nu_{n}$. We also derive some other results on the asymptotic behaviour of the sequence $\left(p_{n}\right)$ and apply some of our results to the theory of fluctuations of random walks. We obtain a necessary and sufficient condition for the first positive ladder epoch to belong to the domain of attraction of a spectrally positive stable law with index $\alpha, \alpha \in(1,2)$.
\end{abstract}

1980 Mathematics subject classification (Amer. Math. Soc.): 670 E 07, $60 \mathrm{~J} 15$.

Keywords and phrases: infinitely divisible sequences, Lévy measure, domain of attraction, fluctuation theory.

\section{Introduction}

This paper is primarily concerned with infinitely divisible distributions $\left(p_{n}\right)$ on the non-negative integers. In this case the Lévy-Hinčin representation takes the form

$$
\hat{p}(z)=\sum_{n \geqslant 0} p_{n} z^{n}=\exp \left\{-\sum_{j \geqslant 1}\left(1-z^{j}\right) \nu_{j}\right\}
$$

C Copyright Australian Mathematical Society 1982 
where $\left(\nu_{j}\right)$ is the Lévy measure and $\lambda=\Sigma \nu_{j}$ is finite. It will be convenient to write (1) in the form

$$
\sum_{n \geqslant 0} p_{n} z^{n}=\exp \left\{-\lambda\left(1-\sum_{j \geqslant 1} \alpha_{j} z^{j}\right)\right\}
$$

so that $\alpha_{j}=\nu_{j} / \lambda$ is a probability measure with $\alpha_{0}=0$. Let $d=$ g.c.d. $\left\{j: \alpha_{j}>0\right\}$. Then $\left(p_{n}\right)$ is supported by the set of multiples of $d$, and $p_{k d}$ is positive for all sufficiently large $k$. Thus, by considering $\bar{\alpha}_{j}=\alpha_{j d} / d$ and $\bar{p}_{n}=p_{n d}$ we may suppose that $d=1$ and that $p_{n}$ is positive for all sufficiently large $n$. This assumption is made throughout this paper. The identity (2) can also be written as the infinite system of equations

$$
n p_{n}=\lambda \sum_{1 \leqslant j \leqslant n} j \alpha_{j} p_{n-j}, \quad n=1,2, \ldots
$$

with $p_{0}=e^{-\lambda}$.

There are a number of results that relate the behaviour of the tails of the measures $\left(p_{n}\right)$ and $\left(v_{n}\right)$. For example Embrechts et al. (1979), Theorem 1 implies the equivalence of the statements:

(i) $\Sigma_{k<[x]} p_{k}$ belongs to $\mathfrak{S}$;

(ii) $\Sigma_{k \leqslant[x]} \alpha_{k}$ belongs to $\delta$; and

(iii) $\Sigma_{k \geq n} p_{k} \sim \lambda \Sigma_{k \geqslant n} \alpha_{k}(n \rightarrow \infty)$.

(Here $\delta$ denotes the class of subexponential distribution functions on $[0, \infty$ ), that is those $F$ satisfying $\left.\lim _{x \rightarrow \infty}\left(1-F^{(2)}(x)\right) /(1-F(x))=2\right)$. The theorem of Embrechts et al., holding for tails of general infinitely divisible probability measures on $[0, \infty)$, generalized work of Cohen (1973), Feller (1971), Pakes (1975) and Teugels (1975).

In this paper our main concern is with the asymptotic behaviour of $p_{n} / \alpha_{n}$, as opposed to that of $\left(\sum_{k \geq n} p_{k}\right) /\left(\sum_{k \geqslant n} \alpha_{k}\right)$. Special cases have already been considered by Hawkes et al. (1978) (in particular in Theorem 3.1). E. M. Wright (1967) works with the system of equations (3) but with the side conditions $p_{0}=1, \lambda=1$ and $\sum \alpha_{i}=\infty$, he proved that the following statements are equivalent:

(i) $p_{n} \sim \alpha_{n}(n \rightarrow \infty)$;

(ii) $\Sigma_{0<i<n} \boldsymbol{\alpha}_{i} \boldsymbol{\alpha}_{n-i}=o(1) \alpha_{n}(n \rightarrow \infty)$; and

(iii) $\Sigma_{0<i<n} p_{i} p_{n-i}=p(1) p_{n}(n \rightarrow \infty)$.

To make the comparison with Wright's work more pertinently, consider $q_{n}=e^{\lambda} p_{n}$, $\beta_{n}=\lambda \alpha_{n}$ hence the recurrence relation (3) becomes

$$
q_{0}=1, \quad n q_{n}=\sum_{j=1}^{n} j \beta_{j} q_{n-j}
$$


as in Wright (1967). Our Theorem 1 gives conditions such that

$$
\lim _{n \rightarrow \infty}\left(q_{n} / \beta_{n}\right)=\exp \left(\sum \beta_{n}\right)
$$

when $\beta_{n+1} / \beta_{n} \rightarrow 1$ and $\Sigma \beta_{n}$ is finite. So all limit values $>1$ are possible in this case, while by Wright (1967) $q_{n} / \beta_{n} \rightarrow 1$ can occur only if $\Sigma \beta_{n}=\infty$. Since Wright's work arose out of an enumeration problem, it was natural to take $q_{0}=1$. While this is not natural in the probabilisitic context, it does emphasize the difference between the two limits (namely $\exp \left(\Sigma \beta_{n}\right)$ and 1 ).

We let $(a * b)_{n}=\Sigma_{0 \leqslant k \leqslant n} a_{k} b_{n-k}$ denote the convolution product of two sequences and $a_{n}^{* k}$ be the $k$ th convolution power of the sequence $\left(a_{n}\right)$. Our principal result is the following:

THEOREM 1. The following three statements are equivalent:

(i) $\alpha_{n}^{* 2} \sim 2 \alpha_{n}$ and $\alpha_{n+1} \sim \alpha_{n}(n \rightarrow \infty)$;

(ii) $p_{n}^{* 2} \sim 2 p_{n}$ and $p_{n+1} \sim p_{n}(n \rightarrow \infty)$; and

(iii) $p_{n} \sim \lambda \alpha_{n}$ and $\alpha_{n+1} \sim \alpha_{n}(n \rightarrow \infty)$.

The convolution condition should be compared with that in the result due to $\mathrm{E}$. M. Wright, quoted above. One consequence of our result is that $\left(p_{n}\right)$ is regularly varying if and only if $\left(\nu_{n}\right)$ is the same. In view of the above result by Embrechts, Goldie and Veraverbeke a similar statement can be made about the tails of the measures $\left(p_{n}\right)$ and $\left(\nu_{n}\right)$. It follows that by constructing a suitable Lévy measure one can produce an example of an infinitely divisible distribution $\left(p_{n}\right)$ such that $\sum_{k \geqslant n} p_{k}$ has regular variation but that $\left(p_{n}\right)$ itself is not regularly varying.

The following (superficially stronger) result is equivalent to that of Theorem 1.

THEOREM 2. Suppose that $R \geqslant 1$. Then the following three statements are equivalent:

(i) $\hat{\alpha}(R)$ is finite, $\alpha_{n}^{* 2} \sim 2 \hat{\alpha}(R) \alpha_{n}$, and $\alpha_{n} \sim R \alpha_{n+1}(n \rightarrow \infty)$;

(ii) $\hat{p}(R)$ is finite, $p_{n}^{* 2} \sim 2 \hat{p}(R) p_{n}$, and $p_{n} \sim R p_{n+1}(n \rightarrow \infty)$; and

(iii) $p_{n} \sim \lambda \hat{p}(R) \alpha_{n}$, and $\alpha_{n} \sim R \alpha_{n+1}(n \rightarrow \infty)$.

Theorem 2 is proved by applying Theorem 1 to the infinitely divisible probability measure $p_{n} R^{n} / \hat{p}(R)$, which corresponds to the Lévy measure $\nu_{j} R^{j}$.

When $\hat{p}(z)$ has radius of convergence $R, R \geqslant 1$, we can use Theorem 2 in the case where $\hat{p}(R)<\infty$. When $\hat{p}(R)=\infty$ we cannot make use of the Chover, Ney and Wainger results. In this situation the results of Hawkes et al. (1978) can be used to give information about the behaviour of $p_{n}$.

To translate the results of that paper to the present situation one lets $b_{n}=$ $R^{n} p_{n} / p_{0}$ and $a_{j}=j R^{j} v_{j}$. One can, for example, apply Theorem 4.2 to obtain the following criteria for $p_{n}$ to be asymptotically geometric. 
LEMMA 1. If $R>1$, each of the following statements implies its successor:

(i) $\Sigma\left(R^{j} \nu_{j}-1 / j\right)$ converges absolutely to $L$;

(ii) $p_{n} \sim p_{0} e^{L} R^{-n}$ as $n \rightarrow \infty$; and

(iii) $\Sigma\left(R^{j} \nu_{j}-1 / j\right)$ converges to $L$.

The following beautiful result, due to Chover, Ney and Wainger plays a crucial rôle in our investigations. Since its proof relies on a deep argument involving Banach algebra techniques it is in some ways unsatisfactory from the point of view of real analysis. We therefore prefer to minimize the number of occasions on which we appeal to it.

Proposition 1. Suppose that the probability measure $\left(\mu_{n}\right)$ satisfies the three conditions:

( $\alpha) \lim _{n \rightarrow \infty} \mu_{n}^{* 2} / \mu_{n}=$ c exists and is finite;

( $\beta) \lim _{n \rightarrow \infty} \mu_{n+1} / \mu_{n}=1 / R$ exists and is positive; and

$(\gamma) d=\hat{\mu}(R)$ is finite.

Assume also that $\Phi(w)$ is a function analytic in a region containing the range of $\hat{\mu}(z)$ for $|z| \leqslant R$.

Then there exists a measure $\left(\Phi(\mu)_{n}\right)$ with

$$
\left(\Phi(\mu) \hat{)}(z) \equiv \sum_{n \geqslant 0} \Phi(\mu)_{n} z^{n}=\Phi(\hat{\mu}(z)) \quad \text { for }|z| \leqslant R,\right.
$$

and for which

$$
\lim _{n \rightarrow \infty} \Phi(\mu)_{n} / \mu_{n}=\Phi^{\prime}(d) .
$$

If moreover $\Phi(w)=\Sigma_{j \geqslant 0} c_{j} w^{j}$ for $|w| \leqslant 1$, where $\Sigma_{j \geqslant 0}\left|c_{j}\right|$ is finite, then we can identify the measure $\Phi(\mu)$ with $\bar{\mu}=\Sigma_{j \geq 0} c_{j} \mu^{* j}$. Finally we must have $c=2 d$ in $(\alpha)$.

For details and examples of sequences satisfying $(\alpha),(\beta)$ and $(\gamma)$ see Chover $e t$ al.(1973a), Theorem 1 and Remark 1. A similar result is proved in Borovkov (1976), Appendix.

\section{Proof of Theorem 1}

For simplicity we will only consider the case where $\lambda=1$. First we show that (iii) implies (ii). Let $N$ be a fixed positive integer and define

$$
I=I(N, n)=\{j: n-N \leqslant j \leqslant n\} .
$$


Then, since $p_{n+1} \sim p_{n}$, we have

$$
\begin{aligned}
\sum_{j \in I} p_{j} p_{n-j} & =p_{n}(1+o(1)) \sum_{j \leqslant N} p_{j} \\
& =p_{n}\left\{1-\sum_{j>N} p_{j}\right\}(1+o(1)) .
\end{aligned}
$$

Hence for some integer $n_{0}=n_{0}(N)$ we have

$$
\left|\sum_{j \in I} p_{j} p_{n-j}-p_{n}\right| \leqslant 2 p_{n} \sum_{j>N} p_{j} \text { for } n \geqslant n_{0} .
$$

Now, by (3),

$$
n p_{n}=\sum j \alpha_{j} p_{n-j}=\left(\sum_{j \in I}+\sum_{j \notin I}\right) j \alpha_{j} p_{n-j}
$$

We have

$$
\begin{aligned}
\sum_{j \in I} j \alpha_{j} p_{n-j} & =n(1+o(1)) \sum_{j \in I} \alpha_{j} p_{n-j} \\
& =n(1+o(1)) \sum_{j \in I} p_{j} p_{n-j} \quad\left(\text { as } \alpha_{n} \sim p_{n}\right) \\
& =n p_{n}(1+o(1))\left\{1-\sum_{j>N} p_{j}\right\}
\end{aligned}
$$

Thus, by (5),

$$
\begin{aligned}
\sum_{j \notin I} j \alpha_{j} p_{n-j} & =n p_{n}(1+o(1)) \sum_{j>N} p_{j} \\
& \leqslant 2 n p_{n} \sum_{j>N} p_{j} \text { for } n \geqslant n_{1}=n_{1}(N) \text { say. }
\end{aligned}
$$

Let $n_{2}$ be such that for $j \geqslant n_{2}$ we have $p_{j} \leqslant 2 \alpha_{j}$. Define

$$
J=J(N, n)=\left\{j: \frac{1}{2} n \leqslant j \leqslant n-N\right\}
$$

so that if $n \geqslant n_{0}+n_{1}+2 n_{2}$

$$
\begin{aligned}
\sum_{j \in J} p_{j} p_{n-j} & \leqslant 2 n^{-1} \sum_{j \in J} j p_{j} p_{n-j} \leqslant 4 n^{-1} \sum_{j \in J} j \alpha_{j} p_{n-j} \\
& \leqslant 4 n^{-1} \sum_{j \notin I} j \alpha_{j} p_{n-j} \\
& \leqslant 8 p_{n} \sum_{j>N} p_{j}, \text { by }(6)
\end{aligned}
$$


If we combine the inequalities (4) and (8) we obtain

$$
\left|p_{n}^{* 2}-2 p_{n}\right| \leqslant 20 p_{n} \sum_{j>N} p_{j}
$$

for sufficiently large $n$. It follows that $p_{n}^{* 2} \sim 2 p_{n}(n \rightarrow \infty)$. Thus (iii) implies (ii).

We now show that (ii) implies (iii). Let $\hat{c}(z)$ be the power series defined by

$$
\hat{c}(z)=(1-\hat{p}(z)) / \hat{p}(z)=\sum_{n \geqslant 0} c_{n} z^{n} .
$$

Then applying Proposition 1 with the function $\Phi(w)=(1-w) / w$ we see that $\Sigma c_{i}$ is absolutely convergent with $\Sigma c_{i}=0, c_{n} \sim-p_{n}(n \rightarrow \infty)$, so that $\left|c_{n}\right| \leqslant 2 p_{n}$ for $n \geqslant n_{3}$, say. It follows from (2) and the definition of $\hat{c}$ that

$$
\hat{\boldsymbol{\alpha}}^{\prime}=\hat{p}^{\prime}+\hat{p}^{\prime} \hat{c} .
$$

Thus if we define $\hat{d}=\hat{p}^{\prime} \hat{c}=\Sigma_{k \geqslant 0} d_{k} z^{k}$ we have

$$
d_{n}=\sum(j+1) p_{j+1} c_{n-j} \text {. }
$$

Now fix $N \geqslant n_{3}$. Then

(9)

$$
\begin{gathered}
\mid \begin{aligned}
\left|\sum_{j \leqslant N}(j+1) p_{j+1} c_{n-j}\right| & \leqslant 2(N+1) \sum_{j \leqslant N} p_{j+1} p_{n-j} \\
= & O(1) p_{n}=o(1) n p_{n}, \\
\left|\sum_{N \leqslant j \leqslant n-N}(j+1) p_{j+1} c_{n-j}\right| & \leqslant 2 n \sum_{N \leqslant j \leqslant n-N} p_{j+1} p_{n-j} \\
& \leqslant 8 n p_{n} \sum_{j>N} p_{j}
\end{aligned}
\end{gathered}
$$

for sufficiently large $n$, and

$$
\begin{aligned}
\left|\sum_{j \in I(N, n)}(j+1) p_{j+1} c_{n-j}\right| & =\left|\sum_{j \in I}\left\{(j+1) p_{j+1}-n p_{n}\right\} c_{n-j}+n p_{n} \sum_{j \leqslant N} c_{j}\right| \\
& \leqslant \sum_{j \in I}\left|(j+1) p_{j+1}-n p_{n}\right|\left|c_{n-j}\right|+n p_{n}\left|\sum_{j \leqslant N} c_{j}\right| \\
& =o(1) n p_{n}+n p_{n}\left|\sum_{j \leqslant N} c_{j}\right| .
\end{aligned}
$$

If we combine (9), (10) and (11) we obtain

$$
\limsup _{n \rightarrow \infty}\left|d_{n}\right| / n p_{n} \leqslant 8 \sum_{j>N} p_{j}+\left|\sum_{j \leqslant N} c_{j}\right| .
$$

As the latter can be made arbitrarily small we have $d_{n}=o(1) n p_{n}$. Since

$$
(n+1) \alpha_{n+1}=(n+1) p_{n+1}+d_{n}
$$


and $p_{n+1} \sim p_{n}$ it follows that $\alpha_{n} \sim p_{n}$, with the result that statements (ii) and (iii) are equivalent. The argument of the first part of the proof shows that (iii), together with its equivalent form (ii), implies (i).

There remains the problem of showing that statement (i) implies (ii). First we state a preliminary lemma.

LemMA 2. Suppose that $\alpha_{n}$ is a probability measure on the non-negative integers such that $\alpha_{n} \sim \alpha_{n+1}$ and $\alpha_{n}^{* 2} \sim 2 \alpha_{n}$ as $n \rightarrow \infty$. Then if $c>1$ there is a positive constant A such that

$$
\alpha_{n}^{* k} \leqslant A c^{k} \alpha_{n} \text { for each } k \text { and } n
$$

The proof of this lemma is very similar to that of Lemma 1 of Chover et al. (1973b), so we just give an outline. If $1<d<c$ we can choose integers $N$ and $K$ such that

$$
\sum_{j \notin I(N, n)} \alpha_{j} \alpha_{n-j} \leqslant d \alpha_{n} \text { if } n \geqslant K .
$$

Next we let $M_{k}=\sup _{n \geqslant 0} \alpha_{n}^{* k} / \alpha_{n}$, define

$$
B=\sup _{0 \leqslant n \leqslant K} \alpha_{n}^{-1}+\sup _{n \geqslant K} \alpha_{n}^{-1} \sum_{j \in I(N, n)} \alpha_{j}
$$

and show that $M_{k+1} \leqslant B+M_{k} d$. Then if $A \geqslant 1+B c /(c-d)$ one sees by induction that $M_{k} \leqslant A c^{k}$.

We now complete the proof of the theorem. It follows from (2) that

$$
p_{n} / \alpha_{n}=e^{-1} \sum_{k \geqslant 1}\left(\alpha_{n}^{* k} / \alpha_{n}\right) / k !
$$

The hypotheses on $\alpha$ imply that $\alpha_{n}^{* k} \sim k \alpha_{n}$ (see Chover et al. (1973a), Lemma 5). Thus by Lemma 2 we can use dominated convergence to deduce that $p_{n} \sim \alpha_{n}$. The Theorem is thus proved.

We remark that the last part of the proof could have been derived by applying Proposition 1 with the measure $\alpha_{n}$ and the function $\Phi(w)=e^{-\lambda(1-w)}$. However our approach has avoided the implicit use of Banach algebra methods. One can in fact use Lemma 2 to show that if $\hat{\Phi}(w)=\Sigma \Phi_{j} w^{j}$ has radius of convergence $R$, $R>1$, then

$$
\Phi(\hat{\alpha}(s))=\sum \psi_{n} s^{n} \quad \text { where } \psi_{n}=\sum \Phi_{k} \alpha_{n}^{* k}
$$

and $\psi_{n} / \alpha_{n} \rightarrow \sum k \Phi_{k}=\Phi^{\prime}(1)$. So the whole situation is much simpler for these functions. This observation does not help us in the second part of the proof where we worked with $\Phi(w)=(1-w) / w$. 
Regarding the conditions in Theorem 1 we have not been able to construct a probability measure $\left(\alpha_{n}\right)$ satisfying $\alpha_{n}^{* 2} \sim 2 \alpha_{n}$ but not $\alpha_{n+1} \sim \alpha_{n}$. Conversely Rudin (1973) gives an example of a log-convex sequence $\left(\alpha_{n}\right)$ with $\sum n \alpha_{n}$ infinite which satisfies $\alpha_{n+1} \sim \alpha_{n}$ but not $\alpha_{n}^{* 2} \sim 2 \alpha_{n}$.

\section{Fluctuation theory}

Let $\left(X_{i}\right)$ be a sequence of independent identically distributed random variables and let $S_{0}=0$ and $S_{n}=\sum_{1<i<n} X_{i}$. We now quote some of the definitions and results from the fluctuation theory of random walks. (For more details the reader should consult Feller (1971).) The first ladder epochs are defined by $L^{+}=\min \left\{k: S_{k}>0\right\}$ and $L^{-}=\min \left\{k \geqslant 1: S_{k} \leqslant 0\right\}$. The number of positive and non-positive terms in the random walk are given by

$$
N_{n}^{+}=\#\left\{k<n: S_{k}>0\right\} \quad \text { and } N_{n}^{-}=\#\left\{k: 1 \leqslant k \leqslant n, S_{k} \leqslant 0\right\}
$$

respectively. If we define

$$
r_{n}=P\left(N_{n}^{+}=n\right)=P\left(L^{-}>n\right), \text { and } q_{n}=P\left(N_{n}^{-}=n\right)=P\left(L^{+}>n\right)
$$

then the generating functions satisfy

$$
\hat{r}(s)=\exp \left\{\sum_{n \geqslant 1}\left(s^{n} / n\right) P\left(S_{n}>0\right)\right\}
$$

and

$$
\hat{q}(s)=\exp \left\{\sum_{n \geqslant 1}\left(s^{n} / n\right) P\left(S_{n} \leqslant 0\right)\right\} .
$$

Also it is known that $P\left(N_{n}^{-}=k\right)=r_{n-k} q_{k}$. Finally the following statements are equivalent:

(i) $E L^{+}<\infty$;

(ii) $\Sigma\left(P\left(S_{n} \leqslant 0\right) / n\right)<\infty$;

(iii) $S_{n}$ drifts to $+\infty$ almost surely; and

(iv) $\sup _{n} N_{n}^{-}<\infty$ almost surely.

We remark that in the case where $E L^{+}$is finite the relation (13) is precisely the equation (2) with $\alpha_{j}=P\left(S_{j} \leqslant 0\right) /\left(j \log E L^{+}\right), \lambda=\log E L^{+}$, and $p_{n}=q_{n} / E L^{+}$. In these circumstances we will be able to apply Theorem 1 .

The following result due to Rogozin (1971) and Spitzer (1956) summarizes the known properties concerning the asymptotic behaviour of the distributions of the random variables $L^{+}$and $N_{n}^{-}$. 
Proposition 2. Suppose that $E L^{+}=\infty$ and that $0<\alpha<1$. Then the following statements are equivalent:

(i) $L^{+}$belongs to the domain of attraction of a spectrally positive stable law with index $\boldsymbol{\alpha}$;

$$
\lim _{n \rightarrow \infty} \frac{1}{n} \sum_{1 \leqslant i \leqslant n} P\left(S_{i} \leqslant 0\right)=1-\alpha
$$

and

$$
\lim _{n \rightarrow \infty} P\left(N_{n}^{-} \leqslant n x\right)=F_{\alpha}(x)
$$

where $F_{\alpha}$ is the generalized arc-sine distribution function (that is the Beta $(\alpha, 1-\alpha)$ distribution).

Rogozin has other results for the case where (14) holds with $\alpha=1$. Doney (1977) has several results on the condition (14) in particular in the case where $\alpha=0$. We now apply our result to obtain more information about the domains of attraction of $L^{+}$.

THEOREM 3. Suppose that $E L^{+}<\infty$ and let $N^{-}=\lim _{n \rightarrow \infty} N_{n}^{-}$. If $1<\alpha<2$ the following statements are equivalent:

(i) $L^{+}$belongs to the domain of attraction of a spectrally positive stable law with index $\boldsymbol{\alpha}$;

(ii) $P\left(S_{n} \leqslant 0\right) \sim n^{1-\alpha} L(n)$ as $n \rightarrow \infty$ where $L$ is a slowly varying function, and

(iii) $N^{-}$belongs to the domain of attraction of a spectrally positive stable law with index $\alpha-1$.

Proof. The equivalence of (i) and (ii) follows by applying Theorem 1 to sequences of regular variation and the necessary and sufficient criteria for a distribution to belong to the domain of attraction of a stable law (see Feller (1971), 574-581 for details).

Since $P\left(N_{n}^{-}=k\right)=r_{n-k} q_{k}$ and $r_{n} \rightarrow\left(E L^{+}\right)^{-1}$ (apply Theorem 4.2 of Hawkes et al. (1978)) we have $P\left(N^{-}=k\right)=q_{k}\left(E L^{+}\right)^{-1}$. As $q_{k}=P\left(L^{+}>k\right)$ is decreasing $P\left(N^{-}>k\right) \sim k^{1-\alpha} L(k)$ is equivalent to

$$
P\left(N^{-}=k\right)=P\left(L^{+}>k\right)\left(E L^{+}\right)^{-1} \sim(\alpha-1) k^{-\alpha} L(k) .
$$

The equivalence of (i) and (iii) follows immediately, and the theorem is proved.

We have not been able to determine a necessary and sufficient condition for $L^{+}$to belong to the domain of attraction of a stable law of index 1. A necessary condition is that (14) holds with $\alpha=1$. There remains the problem of showing that the conditions of our theorem are satisfied in nontrivial situations. 
ExAmple. Suppose that $1<\alpha<2$ and that $\left(Y_{i}\right)$ is a sequence of independent random variables each having the symmetric stable distribution of index $\alpha$. Let $X_{i}=Y_{i}+1$ and let $S_{n}$ be the random walk corresponding to $X_{i}$. Then $\left(S_{n}-n\right) n^{-1 / \alpha}$ has the symmetric stable distribution of index $\alpha$. Thus

$$
\begin{aligned}
P\left(S_{n} \leqslant 0\right) & =P\left(\left(S_{n}-n\right) n^{-1 / \alpha} \leqslant-n^{1-1 / \alpha}\right) \\
& =P\left(Y \leqslant-n^{1-1 / \alpha}\right) \\
& \sim C\left(n^{1-1 / \alpha}\right)^{-\alpha}=C n^{1-\alpha}
\end{aligned}
$$

as $n \rightarrow \infty$ (where $C$ is a positive constant). By the strong law of large numbers $S_{n}$ tends to $+\infty$ and so the theorem is indeed non vacuous.

It is possible to derive other information about the behaviour of the tail $P\left(L^{+}>n\right)$. We now consider random walks generated by $X_{i}$ with $\mu=E X_{1}>0$ and $\operatorname{var} X_{1}=1$. Let $T_{n}=S_{n}-n \mu$. Nagaev (1979) has many results which are sufficient to ensure that

$$
P\left(S_{n} \leqslant 0\right)=P\left(T_{n} \leqslant-n \mu\right) \sim n P(X \leqslant-(n-1) \mu) .
$$

Thus provided in addition $P(X \leqslant-(n-1) \mu)$ has sufficiently smooth asymptotic behaviour (as defined by Theorem 1)

$$
P\left(L^{+}>n\right) \sim E L^{+} P(X \leqslant-(n-1) \mu) \text { as } n \rightarrow \infty .
$$

The reader will be able to show that (15) is valid if

(i) $P(X \leqslant-x) \sim \exp \left(-x^{\alpha}\right)(x \rightarrow \infty), 0<\alpha<1 / 2$, if

(ii) $E|X|^{2+\delta}<\infty$ for some $\delta>0$ and $P(X \leqslant-x) \sim x^{-\alpha} L(x)$ as $x \rightarrow \infty$, where $\alpha>2$ and $L$ is slowly varying; or under some of the other conditions given by Nagaev.

If (15) holds it follows that for each $k \geqslant 1$

$$
E\left(L^{+}\right)^{k}<\infty \text { if and only if } E(X \wedge 1)^{k}<\infty .
$$

Bingham and Goldie (private communication) have shown that (16) always holds provided one has $E X^{2}<\infty$.

\section{Acknowledgement}

This paper was written while the first author was visiting Westfield College, London. He would like to thank the Department of Mathematics for its kind hospitality, and the Royal Society of London and the U. K. Science Research Council for financial support. 


\section{References}

A. A. Borovkov (1976), Stochastic processes in queueing theory (Springer-Verlag, New York).

J. Chover, P. Ney and S. Wainger (1973a), 'Functions of probability measures', J. Analyse Math. 26, 255-302.

J. Chover, P. Ney and S. Wainger (1973b), 'Degeneracy properties of subcritical branching processes', Ann. Probability 1, 663-673.

J. W. Cohen (1973), 'Some results on regular variation for distributions in queueing and fluctuation theory', J. Appl. Probability 10, 343-353.

R. A. Doney (1977), 'A note on a condition satisfied by certain random walks', J. Appl. Probability 14, 843-849.

P. Embrechts, C. M. Goldie and N. Veraverbeke (1979), 'Subexponentiality and infinite divisibility', Z. Wahrscheinlichkeitstheorie und Verw. Gebiete 49, 335-347.

W. Feller (1971), An introduction to probability theory and is applications. Volume I (second edition) (Wiley, New York).

J. Hawkes and J. D. Jenkins (1978), 'Infinitely divisible sequences', Scand. Actuar. J. 2, 65-76.

S. V. Nagaev (1979), 'Large deviations of sums of independent random variables', Ann. Probability 7 , 745-789.

A. G. Pakes (1975), 'On the tails of waiting time distributions', J. Appl. Probability 12, 555-564.

B. A. Rogozin (1971), 'The distribution of the first ladder moment and height and fluctuation of a random walk', Theor. Probability Appl. 16, 575-595.

W. Rudin (1973), 'Limits of ratios of tails of measures', Ann. Probability 1, 982-994.

F. Spitzer (1956), 'A combinatorial lemma and its applications to probability theory', Trans. Amer. Math. Soc. 82, 323-338.

J. L. Teugels (1975), 'The class of subexponential distributions', Ann. Probability 3, 1000-1011.

E. M. Wright (1967), 'A relationship between two sequences', Proc. London Math. Soc. 17, 296-304.

Departement Wiskunde KUL

Celestijnenlaan 200-B

B-3030 Heverlee

Belgium
University College of Swansea

Department of Statistics Singleton Park Swansea SA2 8PP

United Kingdom 